\title{
AN ATYPICAL FORM OF REDUPLICATIVE PARAMNESIA AS THE INAUGURAL PRESENTATION OF DEMENTIA
}

\section{F. Fernandes Martins ${ }^{\star}$, P. Casimiro*, V. Henriques ${ }^{\star}$, C. Fernandes Santos*, N. Descalço*, G. Borges} "Hospital Garcia de Orta, Department of Psychiatry and Mental Health, Almada, Portugal.

\section{Objectives}

To describe an atypical case of reduplicative paramnesia, a delusional misidentification syndrome, in a patient admitted to an inpatient psychiatric unit.

\section{Materials and Methods}

Presentation of a case study, supported by a non-systematic review of the literature with the keywords "misidentification syndrome" and "reduplicative paramnesia".

\section{Background}

- Delusional misidentification syndromes (DMS) are a group of delusional disorders characterized by fixed, false beliefs relating to issues of familiarity ${ }^{1}$, usually characterized by the idea of "doubles" 2 . DMS have been identified in multiple psychiatric and neurological illnesses, presenting in 1-4\% of psychiatric inpatients ${ }^{1}$. They include Capgras syndrome (CS), Fregoli syndrome, intermetamorphosis syndrome and reduplicative paramnesia, among others 5 .

- Reduplicative paramnesia (RP) is most commonly defined as the subjective belief that a place has been duplicated ${ }^{2}$, but it can also be a term for the subjective conviction that a place, person or event is duplicated ${ }^{3}$. Other authors identify a subset of DMS called reduplicative misidentification syndromes ${ }^{2}$ in which RP is included.

\section{Case Presentation}

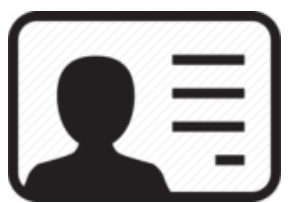

Identification: I.S.C.L,, , 87 years old. No psychiatric background.

Motive of admission to the Psychiatry ward: aggressiveness, secondary to persecutory delusional ideation.

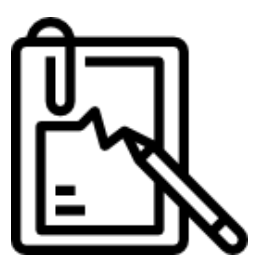

Clinical interview: the patient believed her only son had a twin that had been separated from her at birth, without her knowledge, being reunited with her a few months before the concerned hospital admission. She described having met both her "sons" for a few months, before her "lost son" had a tragic death at the hands of the head of her nursing home. She said the siblings had different personalities.

She also presented with auditory hallucinations and persecutory delusional ideation directed at her daughter-in-law and at the owner of the nursing home where she had been living.

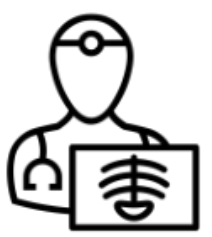

Further investigation: The patient's son denied having a twin brother. A history of what appeared incipient functional loss was taken. The CT-scan showed no significant findings. A detailed neuropsychological examination was consistent with cortical cognitive disturbance involving multiple domains.

\section{Discussion}

- This case presents, most likely, as an uncommon inaugural presentation of dementia, posing a differential diagnosis with late-onset psychosis or organic cause. Further investigation, namely magnetic resonance imaging or electroencephalography, could be useful to rule out other etiologies.

- DMS have been growingly associated with dementia, being relatively common in patients with Alzheimer's Disease and Dementia with Lewy Bodies ${ }^{4}$. They are often associated with violent acts ${ }^{4,5}$, which is also illustrated in this case.

- The DMS here reported constitutes an atypical form of RP, which most frequently refers to duplicated places. RP is most commonly associated with neurological disorders, unlike other DMS ${ }^{2,4}$.We chose to classify this presentation as RP as it doesn't fit the description of CS (in which a familiar person is thought to have been replaced by an impostor) or of a clonal pluralization syndrome (in which the self or others are duplicated as exact same copies, including in personality). It is, instead, a rare phenomenon of reduplication rarely described in literature. ${ }^{6}$

\section{Conclusions}

